\title{
Expression of DNA reparation genes in anhydrobiotic insect Polypedilum vanderplanki
}

\author{
Alexander Nesmelov \\ IFMB KFU, Kazan, Russia \\ nesmelov@gmail.com
}

\author{
Sabina Kondratyeva \\ IFMB KFU, Kazan, Russia \\ sabinakondr@gmail.com
}

\author{
Taisiya Voronina \\ IFMB KFU, Kazan, Russia \\ vorotaisiya@gmail.com
}

\begin{abstract}
Anhydrobiosis is survival of complete body desiccation, tightly linked to the ability to cope with a massive DNA damage. However, in anhydrobiotic midge Polypedilum vanderplanki this ability is not accompanied by a huge induction of genes of DNA reparation system which is typical for many other anhydrobiosis-related genes in this insect.
\end{abstract}

Keywords - anhydrobiosis, Polypedilum vanderplanki, DNA reparation, gene expression

\section{Motivation and aim}

Motivation

$P$. vanderplanki is the most complex animal able to survive body desiccation. Its ability to cope with extraordinary DNA damage is an interesting phenomenon for basic science and may serve as a model for the development of dry preservation technologies.

Aim

Earlier we have shown a presence of massive DNA damage in $P$. vanderplanki, performed genome assembly and gene annotation in this insect $[1,2]$. Here we aimed to identify genes of different DNA reparation systems known for Drosophila melanogaster and reveal what are the DNA reparation pathways most activated in response to desiccation.

\section{Methods}

We extended existing gene annotation in $P$. vanderplanki by the additional search for genes of DNA reparation system. We identified orthologs of the genes of different DNA reparation pathways known for $\mathrm{D}$. melanogaster using OrthoFinder 2.2.7. Gene models for identified
$P$. vanderplanki orthologs were estimated using Nanopore reads and EST database. Using RNA-seq data, we studied the expression of identified DNA reparation genes in $P$. vanderplanki in response to desiccation and across different life stages. Employing WGCNA analysis, we also analyzed the membership of genes of DNA reparation system in different modules of the gene coexpression network.

\section{Results}

In $P$. vanderplanki DNA reparation genes are typically expressed at a lower level and are less induced in response to desiccation, in comparison to previously identified genes related to anhydrobiosis. Most of these genes are not included in the "key" module of gene network associated with anhydrobiosis. Moreover, most of them are not assigned to any existing module, suggesting that their regulation is perturbed during anhydrobiosis induction. Among different DNA reparation subsystems, nucleotide excision repair seems to be most activated since it contains highest number of genes, significantly upregulated on a course of anhydrobiosis.

\section{ACKNOWLEDGMENT}

Supported by the Russian Science Foundation grant No. 19-74-00133.

\section{REFERENCES}

[1] Gusev O.A. et al. (2010) Anhydrobiosis-associated nuclear DNA damage and repair in the sleeping chironomid: linkage with radioresistance. PLoS One. 5(11):e14008

[2] Gusev O.A. et al. (2014) Comparative genome sequencing reveals genomic signature of extreme desiccation tolerance in the anhydrobiotic midge. Nature Communications 5:4784 